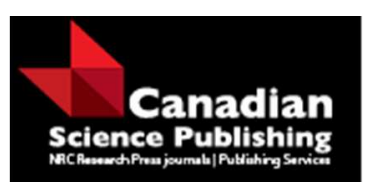

Canadian Journal of Physics

Revue canadienne de physique

\title{
A modified holographic Ricci dark energy model in a self- creation cosmology
}

\begin{tabular}{|r|l|}
\hline Journal: & Canadian Journal of Physics \\
\hline Manuscript ID & cjp-2016-0486.R1 \\
\hline Manuscript Type: & Article \\
\hline Date Submitted by the Author: & $16-$ Aug-2016 \\
\hline Complete List of Authors: & $\begin{array}{l}\text { Bhaskar Rao, M.P.V.V.; Vignan's Institute of Information Technology } \\
\text { REDDY, DRK; Andhra University, Waltair, Mathematics } \\
\text { Babu, K. Sobhan; JNTU K College of Engg. Narsaraopeta }\end{array}$ \\
\hline Keyword: & $\begin{array}{l}\text { Self-creation cosmology, holographic dark energy, Modified Ricci dark } \\
\text { energy, Cosmology, Bianchi type-II model }\end{array}$ \\
\hline \multicolumn{2}{|c}{} \\
\hline
\end{tabular}

SCHOLARONE ${ }^{\text {m }}$

Manuscripts 


\title{
A modified holographic Ricci dark energy model in a self-creation cosmology
}

\author{
M.P.V.V. Bhaskara Rao ${ }^{1}$, D.R.K. Reddy ${ }^{2 *}$, K. Sobhan Babu ${ }^{3}$ \\ ${ }^{1}$ Dept. of Basic Sciences and Humanities, Vignan's Institute of Information Technology, \\ Duvvada, Visakhapatnam, India. \\ ${ }^{2}$ Dept. of Applied Mathematics, Andhra University, Visakhapatnam, India. \\ ${ }^{3}$ Dept. of Mathematics, JNTU K College of Engg. Narsaraopeta, India.
} reddy_einstein@yahoo.com

\begin{abstract}
This paper deals with a locally rotationally symmetric Bianchi type -II space-time with dark matter and anisotropic modified holographic Ricci dark energy as source in second self-creation theory of gravitation proposed by Barber (Gen. Relativ. Gravit.14: 117, 1982).To solve the field equations of this theory we have used (i) hybrid expansion law, (ii) a relation between metric potentials and a modified holographic Ricci dark energy given by Chen and Jing (Phys. Lett. B 679: 144, 2009). The solution obtained represents a Bianchi type-II modified holographic dark energy model in self-creation cosmology. We observe that there is a smooth transition of the universe from decelerated phase to accelerated phase. This fact is in good agreement with the observations of modern cosmology. We have also discussed some important physical aspects of the model.
\end{abstract}

Keywords: Self-creation cosmology, holographic dark energy, Modified Ricci dark energy, Cosmology, Bianchi type-II model.

\section{Introduction}

Observations of modern cosmology [1-2] confirm that our universe is in a state of accelerated expansion. It is believed that this cosmic acceleration is due to the exotic negative pressure in the universe and is known as dark energy. However, the exact information about this is not yet very clear. Dark energy is usually described by the equation of state (EoS) parameter given by $\omega=p_{\Lambda} / \rho_{\Lambda}$, where $\mathrm{p}_{\Lambda}$ is the dark energy pressure and $\rho_{\Lambda}$ dark energy density, which is not constant. There are two ways of explaining dark energy either by considering the dark energy candidates such as cosmological constant $\Lambda$, quintessence, phantom, k-essence, quintom models or by constructing dark energy models using 'modified gravity approach'. Important 
modifications of Einstein's theory of gravity are $f(R)$ and $f(R, T)$ theories of gravity [3-4] (R is the Ricci scalar and $\mathrm{T}$ is the trace of energy tensor) and scalar-tensor theories of gravitation formulated by Brans and Dicke [5], Barber [6] and Saez and Ballester [7].

In Brans-Dicke (BD) theory, in addition to the usual metric tensor field $g_{\mathrm{ij}}$, a scalar field $\phi$, which plays the role of inverse of the gravitational constant, has been introduced. Subsequently Barber [6] has formulated two self- creation theories which modify BD theory and general relativity. In this mass of the universe is supposed to be created out of self contained gravitational, scalar and matter fields. Out of these two theories, the first theory violates [8] equivalence principle and hence is not satisfactory. The second theory is a modification of general relativity to a variable $G$ theory in which the scalar field $\phi$ does not gravitate, but simply divides the matter tensor acting as a reciprocal of gravitational constant. Also the scalar field couples to the trace of energy momentum tensor. The field equations in Barber's [6] second self - creation theory of gravitation are given by

$$
R_{i j}-\frac{1}{2} g_{i j} R=-8 \pi \phi^{-1} T_{i j}
$$

and

$$
\square \phi=\phi_{; k}^{k}=\frac{8 \pi}{3} \mu T
$$

where $\mu$ is a coupling constant to be determined from experiments (the measurement of the deflection of light restricts the value of coupling constant to $|\mu| \leq 10^{-1}$ ), $T_{i j}$ is the energy momentum tensor, $G=\phi^{-1}, T$ is the trace of the energy momentum tensor, i.e. $T=T_{i}^{i}$ and the other symbols have their usual meaning. Also, the usual conservation of energy is not valid in this particular theory.

Investigation of holographic dark energy models to explain the cosmic acceleration is, recently, attracting the attention of several research workers in this particular field. Holographic dark energy is based on holographic principle which states that in quantum gravity, the entropy of a system scales not with its volume but with its surface area $\mathrm{L}^{2}$. Cohen et al. [9] motivated by this principle suggested that the vacuum energy density is proportional to the Hubble scale $L \approx H^{-1}$. Li [10] has defined the energy density of holographic dark energy as 


$$
\rho_{\Lambda}=3 c^{2} M_{p l}^{2} L^{-2}
$$

where $\mathrm{L}$ is the infrared IR cut off radius, $\mathrm{c}$ is a constant and

$$
M_{p l}^{2}=\frac{1}{8 \pi G}
$$

is the Planck mass. The IR cut off has been considered as the Hubble radius or future event horizon. Later Gao et al. [11] have assumed that the future event horizon is replaced by the inverse of the Ricci scalar curvature i.e. $L \approx|R|^{-\frac{1}{2}}$. In this case the model is called Ricci dark energy model. Also Granda and Oliveros [12-13] proposed a new holographic Ricci dark energy model with energy density given by

$$
\rho_{\Lambda}=3 M_{p l}^{2}\left(\alpha H^{2}+\beta \dot{H}\right)
$$

and subsequently Chen and Jing[14] have modified this model with energy density given by

$$
\rho_{\Lambda}=3 M_{p l}^{2}\left(\alpha H^{2}+\beta \dot{H}+\eta \ddot{H} H^{-1}\right)
$$

where an overhead dot indicates differentiation with respect to t. The model with this energy density is known as modified Ricci dark energy model.

In recent years, there has been a lot of interest in the discussion of the dynamics of holographic dark energy models. Several authors have discussed different aspects of holographic dark energy[ 9, 15-16]. Holographic dark energy models in BD theory have been studied by many authors[17-20]. Also, Sarkar and Mahanta [21] and Sarkar [22-24] have investigated minimally interacting holographic dark energy models with linearly varying deceleration parameter in Bianchi type-I and V universes and interacting holographic dark energy models in Bianchi type-II space-time respectively. Adhav et al. [25-26] have discussed interacting holographic dark energy models in Bianchi type-I and V space-times respectively.

Kiran et al. [27-29], Reddy et al. [30] and Umadevi and Ramesh [31] have obtained minimally interacting holographic dark energy models in BD and Saez-Ballester scalar-tensor theories of gravitation. Very recently, Das and Sultana [32] presented an anisotropic Bianchi type-II modified holographic Ricci dark energy cosmological model, in general relativity, with hybrid expansion law.

The above investigations motivated us to discuss, in this paper, the anisotropic locally rotationally symmetric Bianchi type-II modified holographic Ricci dark energy model in the second self -creation theory of gravitation proposed by Barber [6]. It is well known that the 
study of spatially homogeneous and anisotropic cosmological model is significant to understand the early stages of evolution of the universe .The plan of this paper is as follows: the metric and field equations are given in Sect.2. In Sect.3, we present the solution of field equations using hybrid expansion law. Sect.4 is devoted to the physical properties of the model obtained. The last Sect. contains some concluding remarks.

\section{Metric and field equations}

We consider the locally rotationally symmetric (LRS) Bianchi type space-time in the form

$$
d s^{2}=-d t^{2}+A^{2}\left(d x^{2}+d z^{2}\right)+B^{2}(d y+x d z)^{2}
$$

where $\mathrm{A}$ and $\mathrm{B}$ are functions of cosmic time $\mathrm{t}$ only.

The energy momentum tensors for pressure less matter and holographic dark energy are, respectively, given by

$$
\begin{aligned}
& T_{i j}=\rho_{m} u_{i} u_{j}, \quad i, j=1,2,3,4 \\
& \bar{T}_{i j}=\left(\rho_{\Lambda}+p_{\Lambda}\right) u_{i} u_{j}+g_{i j} p_{\Lambda}
\end{aligned}
$$

where $\rho_{m}$ is matter energy density, $\rho_{\Lambda}$ is the energy density of the modified holographic Ricci dark energy. In this particular case, Barber's field equations (1) and (2) take the form

$$
\begin{aligned}
& R_{i j}-\frac{1}{2} g_{i j} R=-8 \pi \phi^{-1}\left(T_{i j}+\overline{T_{i j}}\right) \\
& \square \phi=\phi_{; k}^{, k}=\frac{8 \pi}{3} \mu(T+\bar{T})
\end{aligned}
$$

Now, parameterising, we have, from Eq. (8)

$$
\bar{T}_{i}^{j}=\operatorname{diag}\left[-1, \omega_{x}, \omega_{y}, \omega_{z}\right] \rho_{\Lambda}=\operatorname{diag}[-1, \omega,(\omega+\delta),(\omega+\gamma)] \rho_{\Lambda}
$$

Here we have used the EoS parameter given by

$$
\omega \rho_{\Lambda}=p_{\Lambda}
$$

and $\omega_{x}, \omega_{y}, \omega_{z}$ are the directional EoS parameters along $x, y, z$ axes respectively. For the sake of simplicity we choose $\omega_{x}=\omega$ and the skewness parameters $\delta$ and $\gamma$ are the deviations from $\omega$ on $y$ and $z$ axes respectively.

Now using co moving coordinate system, the field equations (9)and (10), with the help of Eq.(11), for the metric given by Eq.(7) take the form 


$$
\begin{gathered}
\frac{\ddot{B}}{B}+\frac{\ddot{A}}{A}+\frac{\dot{A} \dot{B}}{A B}+\frac{1}{4} \frac{B^{2}}{A^{4}}=-8 \pi \phi^{-1} \omega \rho_{\Lambda} \\
2 \frac{\ddot{A}}{A}+\left(\frac{\dot{A}}{A}\right)^{2}-\frac{3}{4} \frac{B^{2}}{A^{4}}=-8 \pi \phi^{-1}(\omega+\delta) \rho_{\Lambda} \\
2 \frac{\ddot{A}}{A}+\left(\frac{\dot{A}}{A}\right)^{2}-\frac{3}{4} \frac{B^{2}}{A^{4}}=-8 \pi \phi^{-1}(\omega+\gamma) \rho_{\Lambda} \\
2 \frac{\dot{A}}{A} \frac{\dot{B}}{B}+\left(\frac{\dot{A}}{A}\right)^{2}-\frac{1}{4} \frac{B^{2}}{A^{4}}=8 \pi \phi^{-1}\left(\rho_{\Lambda}+\rho_{m}\right) \\
\ddot{\phi}+\dot{\phi}\left(2 \frac{\dot{A}}{A}+\frac{\dot{B}}{B}\right)=\frac{8 \pi}{3} \mu(T+\bar{T})
\end{gathered}
$$

We ,now, define the kinematical and physical parameters of cosmology which will be useful to solve the field equations.

The spatial volume of the space-time (7) is given by

$$
V=a^{3}(t)=A^{2} B
$$

where $a(t)$ is the scale factor of the universe.

The average Hubble parameter is

$$
H=\frac{1}{3}\left(2 \frac{\dot{A}}{A}+\frac{\dot{B}}{B}\right)
$$

The scalar expansion is given by

$$
\theta=2 \frac{\dot{A}}{A}+\frac{\dot{B}}{B}
$$

The shear scalar is defined as

$$
\sigma^{2}=\frac{1}{3}\left(\frac{\dot{A}}{A}-\frac{\dot{B}}{B}\right)^{2}
$$

The anisotropy parameter is given by

$$
\Delta=\frac{1}{3} \sum_{i=1}^{3}\left(\frac{H_{i}-H}{H}\right)^{2}
$$

where $H_{i}, i=1,2,3$ are directional Hubble parameters. 


\section{Solution of the field equations and the model}

Equations (14) and (15), immediately, yield

$$
\gamma=\delta
$$

Using Eq.(23) the field equations (13)-(17) reduce to a system of four independent equations in seven unknowns, A, B, $\rho_{\mathrm{m}}, \rho_{\Lambda}, \phi, \delta, \omega$. Hence to find a determinate solution of the system we require three more conditions. We use the following physically significant conditions:

(i) The shear scalar $\sigma^{2}$ is proportional to expansion scalar $\theta$ so that we have [33]

$$
\mathrm{A}=\mathrm{B}^{\mathrm{n}}
$$

where $n \neq 0$ is a positive constant which takes care of anisotropy of the space-time.

(ii) We consider the average scale factor as a combination of power law and exponential law, given by Akarsu et al. [34], as

$$
a(t)=a_{0} t^{\alpha_{1}} e^{\alpha_{2} t}
$$

where $\alpha_{1}$ and $\alpha_{2}$ are non-negative constants. Here, when $\alpha_{1}=0$ we get the exponential law and when $\alpha_{2}=0$ we obtain power law. Thus, Eq. (25) gives the combination of exponential and power law which is usually known as hybrid expansion law. This choice of average scale factor leads to a time dependent deceleration parameter. The solution gives inflation and radiation dominance era with subsequent transition from decelerating to accelerating phase of the universe.

(iii) The energy density of modified holographic Ricci dark energy, in view of Eqs (4) and (6) and because of the fact that $\phi$ plays the role of $\mathrm{G}^{-1}$, is given by the equation (Chen and Jing [35]).

$$
\rho_{\Lambda}=\frac{3 \phi}{8 \pi}\left(\alpha H^{2}+\beta \dot{H}+\eta \ddot{H} H^{-1}\right)
$$

Also, since the field equations are highly non-linear, we use the extra physically valid condition $[28,36]$

$$
(T+\bar{T})=0
$$


which physically corresponds to the vanishing of the trace of both dark matter and dark energy tensors. However, the problem of over determinacy is settled by the satisfaction of the field equations with the solution obtained.

Now from Eqs. (18), (24) and (25) we obtain the metric potentials as

$$
A=\left(a_{0} t^{\alpha_{1}} e^{\alpha_{2} t}\right)^{\frac{3 n}{2 n+1}}, \quad B=\left(a_{0} t^{\alpha_{1}} e^{\alpha_{2} t}\right)^{\frac{3}{2 n+1}}
$$

Using Eq.(28), we can write the metric (7) in the form

$$
d s^{2}=-d t^{2}+\left(a_{0} t^{\alpha_{1}} e^{\alpha_{2} t}\right)^{\frac{6 n}{2 n+1}}\left(d x^{2}+d z^{2}\right)+\left(a_{0} t^{\alpha_{1}} e^{\alpha_{2} t}\right)^{\frac{6}{2 n+1}}(d y+x d z)^{2}
$$

Also, from Eqs.(17),(27) and (28) we have the scalar field given by

$$
\phi=\varphi_{0} \int\left(a_{0} t^{\alpha_{1}} e^{\alpha_{2} t}\right)^{-3} d t+\psi_{0}
$$

where $\varphi_{0}$ and $\psi_{0}$ are constants of integration which can be set as $\varphi_{0}=1$ and $\psi_{0}=0$ (the integral in Eq. (30) can be evaluated using incomplete gamma function).

\section{Physical and kinematical properties of the model}

Bianchi type-II modified holographic Ricci dark energy universe in self-creation cosmology is, now, represented by the space-time given by the Eq. (29) with the scalar field given by Eq. (30).

We shall now compute the physical and kinematical parameters in the universe (29) which play an important role in the discussion of cosmology.

The spatial volume of the universe is

$$
V=\left(a_{0} t^{\alpha_{1}} e^{\alpha_{2} t}\right)^{3}
$$

The average Hubble parameter is

$$
H=\left(\frac{\alpha_{1}}{t}+\alpha_{2}\right)
$$

The scalar expansion in the universe is

$$
\theta=3\left(\frac{\alpha_{1}}{t}+\alpha_{2}\right)
$$

The shear scalar is 


$$
\sigma^{2}=\frac{3(n-1)^{2}}{(2 n+1)^{2}}\left(\frac{\alpha_{1}}{t}+\alpha_{2}\right)^{2}
$$

The anisotropy parameter is

$$
\Delta=\frac{4}{3}\left(\frac{\mathrm{n}-1}{2 \mathrm{n}+1}\right)^{2}
$$

From the equations (26) and (32) the energy density of modified holographic dark energy in the universe is obtained as

$$
\rho_{\Lambda}=\frac{3 \phi}{8 \pi \mathrm{t}^{2}}\left[\alpha\left(\alpha_{1}+\alpha_{2} \mathrm{t}\right)^{2}-\beta \alpha_{1}+2 \eta \alpha_{1}\left(\alpha_{1}+\alpha_{2} \mathrm{t}\right)^{-1}\right]
$$

where $\phi$ is given by Eq.(30).

From the Eqs. (13),(28) and (36) we get the EoS parameter in the model as

$$
\omega=\frac{-\left\{\frac{9\left(\alpha_{1}+\alpha_{2} t\right)^{2}\left(n^{2}+n+1\right)}{(2 n+1)^{2} t^{2}}-\frac{3 \alpha_{1}(n+1)}{(2 n+1) t^{2}}-\frac{1}{4}\left(a_{0} t^{\alpha_{1}} e^{\alpha_{2} t}\right)^{-\frac{6(2 n-1)}{2 n+1}}\right\}}{\frac{3}{t^{2}}\left[\alpha\left(\alpha_{1}+\alpha_{2} t\right)^{2}-\beta \alpha_{1}+2 \eta \alpha_{1}\left(\alpha_{1}+\alpha_{2} t\right)^{-1}\right]}
$$

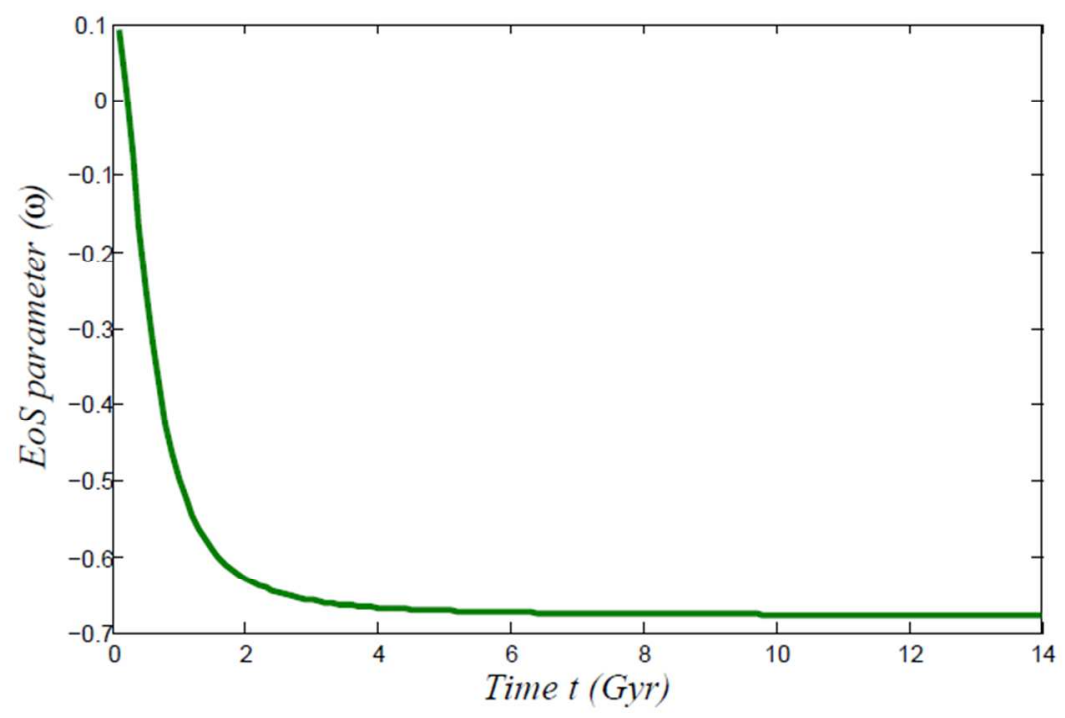

Fig. 1: Plot of EoS parameter versus time $\mathrm{t}$ for $n=0.95$

$$
a_{0}=1, \alpha_{1}=0.3, \alpha_{2}=0.7, \alpha=1.5, \beta=0.5 \text { and } \eta=0.4 \text {. }
$$

From the Eqs. (13), (14), (23), (28) and (36) the skewness parameters are given by 


$$
\delta=\gamma=\frac{\left\{\frac{9\left(\alpha_{1}+\alpha_{2} t\right)^{2}\left(1+n-2 n^{2}\right)}{(2 n+1)^{2}}+\frac{3 \alpha_{1}(n-1)}{(2 n+1)}+t^{2}\left(a_{0} t^{\alpha_{1}} e^{\alpha_{2} t}\right)^{-\frac{6(2 n-1)}{2 n+1}}\right\}}{3\left[\alpha\left(\alpha_{1}+\alpha_{2} t\right)^{2}-\beta \alpha_{1}+2 \eta \alpha_{1}\left(\alpha_{1}+\alpha_{2} t\right)^{-1}\right]}
$$

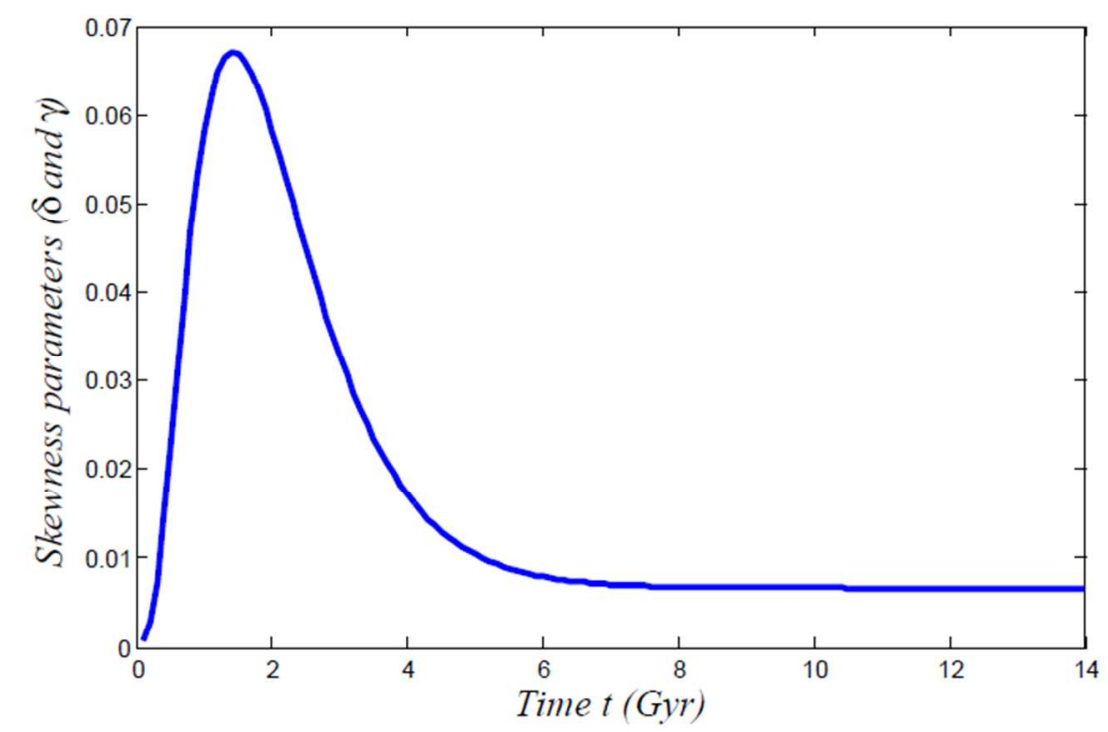

Fig. 2: Plot of skewness parameters versus time $t$ for $n=0.95$

$$
a_{0}=1, \alpha_{1}=0.3, \alpha_{2}=0.7, \alpha=1.5, \beta=0.5 \text { and } \eta=0.4 \text {. }
$$

The energy density of matter can be obtained from the Eqs. (16), (28) and (36) as

$\rho_{m}=\frac{3 \phi}{8 \pi t^{2}}\left\{\left(\frac{3 n(n+2)}{(2 n+1)^{2}}-\alpha\right)\left(\alpha_{1}+\alpha_{2} t\right)^{2}+\beta \alpha_{1}-2 \eta \alpha_{1}\left(\alpha_{1}+\alpha_{2} t\right)^{-1}-\frac{1}{12}\left(a_{0} t^{\alpha_{1}} e^{\alpha_{2} t}\right)^{\frac{-6(2 n-1)}{2 n+1}}\right\}$

where $\phi$ is given by Eq. (30).

The matter density parameter $\Omega_{m}$ and modified holographic Ricci dark energy density parameter $\Omega_{\Lambda}$ are given by

$\Omega_{m}=\frac{\rho_{m}}{3 H^{2}}, \Omega_{\Lambda}=\frac{\rho_{\Lambda}}{3 H^{2}}$

Using equations (32), (36), (39) in the above equation we get the overall density parameter as

$\Omega=\frac{\phi}{8 \pi}\left\{\frac{3 n(n+2)}{(2 n+1)^{2}}-\frac{t^{2}}{12} \frac{\left(a_{0} t^{\alpha_{1}} e^{\alpha_{2} t}\right)^{\frac{-6(2 n-1)}{2 n+1}}}{\left(\alpha_{1}+\alpha_{2} t\right)^{2}}\right\}$

where $\phi$ is given by Eq.(30) 
Using Eq. (32), the deceleration parameter is obtained as

$$
q=\frac{d}{d t}\left(\frac{1}{H}\right)-1=-1+\frac{\alpha_{1}}{\left(\alpha_{1}+\alpha_{2} t\right)^{2}}
$$

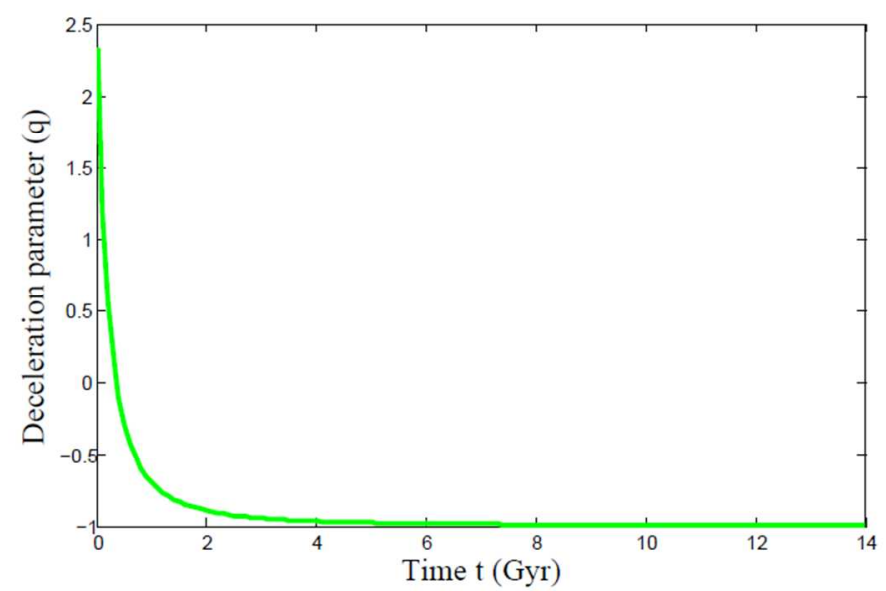

Fig. 3: Plot of deceleration parameter versus time $t$ for $\alpha_{1}=0.3$ and $\alpha_{2}=0.7$.

To describe models close to $\Lambda C D M$, cosmic jerk parameter $j$ is used in cosmology. It is defined as the third derivative of the scale factor with respect to the cosmic time and is given by (Chiba and Nakamura [37])

$$
j(t)=\frac{1}{H^{3}} \frac{\dddot{a}}{a}=q+2 q^{2}-\frac{\dot{q}}{H}
$$

Cosmologists believe that deceleration to acceleration transition of the universe occurs for models with positive value of jerk parameter and negative value of deceleration parameter. The jerk parameter for $\Lambda C D M$ model have a constant jerk, $j=1$. In this model, we get

$$
j(t)=1-\frac{3 \alpha_{1}}{\left(\alpha_{1}+\alpha_{2} t\right)^{2}}+\frac{2 \alpha_{1}}{\left(\alpha_{1}+\alpha_{2} t\right)^{3}}
$$




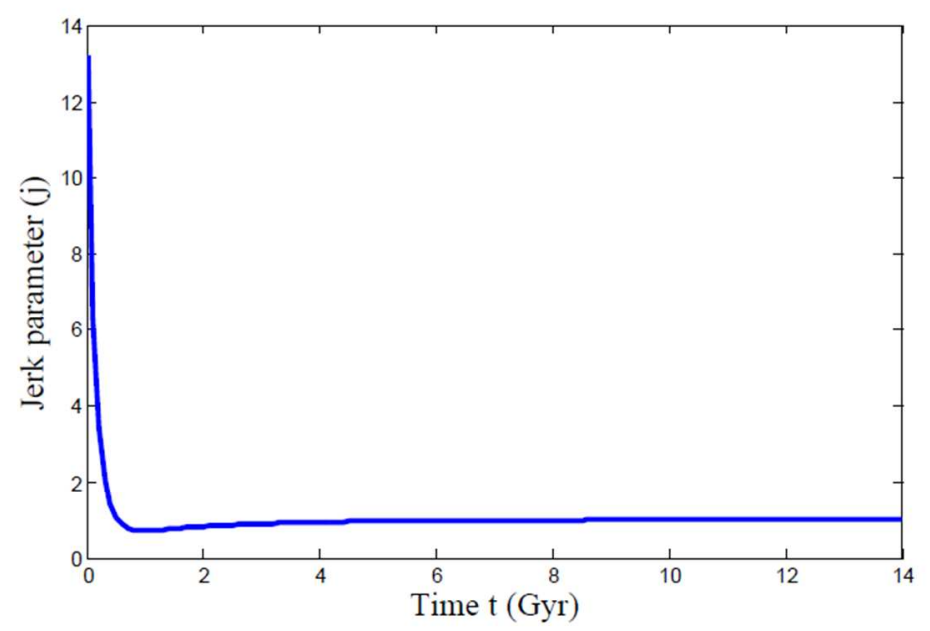

Fig. 4: Plot of jerk parameter versus time $t$ for $\alpha_{1}=0.3$ and $\alpha_{2}=0.7$.

The above results facilitate the discussion of the physical behavior of the model (29). It may be observed that the spatial volume increases with time. This confirms the spatial expansion of the universe. It can be seen that all the above physical and kinematical parameters of the model diverse at $\mathrm{t}=0$ while all of them vanish as $\mathrm{t}$ approaches infinity. It may also be noted that when $n=1$, the anisotropy parameter vanishes and the model becomes isotropic and shear free at late times.

Figure 1 shows the behavior of EoS parameter of dark energy versus cosmic time $t$. It can be seen that the universe evolves through the dust region and attains a constant value in the quintessence region. Figure 2 gives the variation of skewness parameter with time $t$. It can be seen that at late times it approaches almost zero. The behavior of deceleration parameter is described by Fig. 3. It is observed that there occurs a smooth transition from early decelerated phase to late time acceleration. Fig. 4 shows variation of jerk parameter with time $t$. It can be seen that it is positive throughout the history of the universe and becomes $\Lambda C D M$ model at late times. These results are in good agreement with modern observations of cosmology.

\section{Concluding remarks}

In this paper, we have investigated a spatially homogeneous LRS Bianchi type-II modified holographic Ricci dark energy model in Barber's [6] second self-creation cosmology. We have used a hybrid expansion law and some viable physical conditions to find a determinate solution of the field equations. Using the solution we have presented the model of the universe in this 
theory. We have also studied the physical and kinematical properties of the universe. It is observed that the spatial volume increases with time. It may be noted that anisotropy and skewness parameters of the universe approach zero at later stage of the universe. It is interesting to note that when $n=1$, the universe becomes isotropic and shear free. Also, it can be seen that a smooth transition of the universe takes place from decelerated phase to accelerated phase since the deceleration parameter is negative and the cosmic jerk parameter is positive throughout the history of the universe. Hence our results are in accordance with the recent observational data.

Acknowledgements: The authors are grateful to the reviewer for constructive comments which have helped a lot to improve the presentation of the manuscript.

\section{References}

[1] Riess, A.G., et al.: Astron J.116,1009 (1998)

[2] Perlmutter, S., et al.: Nature 391,51 (1998)

[3] Caroll, Sm., et al.: Phy. Rev. D 75, 24014 (2007)

[4] Harko,T.et al.: Phy.Rev.D84,024020(2011)

[5] Brans, C., Dicke, R.H.: Phys. Rev. 124, 925 (1961)

[6] Barber, G.A.: Gen. Relativ. Gravit. 14, 117 (1982).

[7] Saez, D., Ballester, V.J.: Phys. Lett. A 113, 467 (1986)

[8] Brans, C.: Gen Relativ. Gravit. 19,1949 (1987)

[9] Cohen, A., et al.: Phys. Rev. Lett. 82, 4971 (1999)

[10] Li, M.: Phys. Lett. B 603,1 (2004)

[11] Gao, C. et al.: Phys.Rev.D79, 043511 (2009)

[12] Granda, L.N., Oliveros, A.: Phys.Lett.B 669, 275 (2008)

[13] Granda, L.N., Oliveros, A.: Phys. Lett. B 671, 199 (2009)

[14] Chen,S., Jing,J.: Phys. Lett. B 679,144 (2009)

[15] Horova,P. Minic, D.: Phys. Rev. Lett. 85,1610 (2000)

[16] Hsu, S.D.H.: Phy. Lett. B 594 ,13 (2004)

[17] Thomas, S.: Phys. Rev. Lett. 89, 081301 (2002)

[18] Setare, M. R.: Phys. Lett. B 644, 99 (2007)

[19] Setare, M. R., Vagenas, E.C.: Int. J. Mod. Phys. D18, 147 (2009) 
[20] Sheykhi, A.: arxiv: 0907. 5458v4 [hep-th] (2009)

[21] Sarkar, S; Mahanta, C. R.; Int. J. Theor. Phys. 52, 1482 (2013)

[22] Das, S., Mamon, A.A.: Astrophys. Space sci. 351, 651 (2014)

[23] Sarkar, S.: Astrophys. Space Sci. 349, 985 (2014b)

[24] Sarkar, S.: Astrophys. Space Sci. 352, 859 (2014a)

[25] Adhav, K. S. et al.; Astrophys. Space Sci. 353, 249 (2014)

[26] Adhav, K. S. et al.; Astrophys. Space Sci. 359:24(2015)

[27] Kiran, M., et al.: Astrophys. Space Sci. 354, 577 (2014)

[28] Kiran, M., et al.: Astrophys. Space Sci. 356, 407 (2015a)

[29] Kiran, M., et al.: Astrophys. Space Sci. 360, 54 (2015b)

[30] Reddy, D.R.K., et al.: Prespace time J. 7, 100 (2016)

[31] Umadevi, S., Ramesh, G.: Astrophys. Space Sci. 359, 51 (2015)

[32] Das, K., Sultana, T.: Astrophys. Space Sci.360:4(2015)

[33] Collins, C. B. et al.: Gen.Relativ. Gravit.12,805(1983)

[34] Akarsu, O., et al.: J. csmol. Astropart. Phys. 01,022(2014)

[35] Chen, S., Jing, J.: Phys. Lett. B 679, 144 (2009)

[36] Vidyasagar, T., et al.: Eur. Phys. J. Plus,129,36(2014)

[37] Chiba, T., Nakamura,T.: Prog.Theor.Phys. 100,1077(1998) 